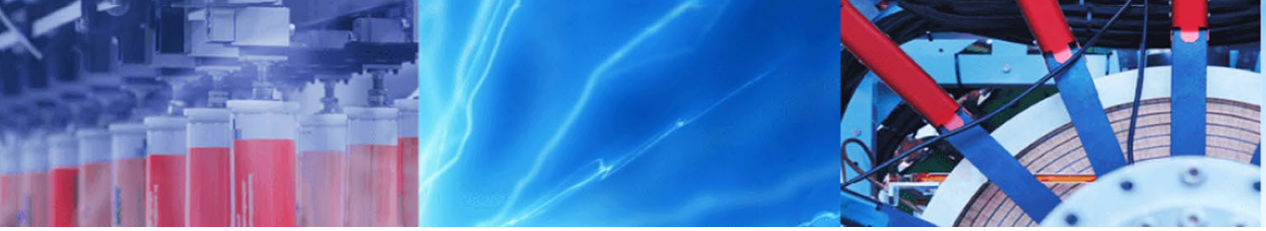

Research Article

\title{
Application of improved data-driven diagnostics workflow based on coupled hydro-mechanical model for stimulation candidate selection in a complex carbonate reservoir
}

\author{
Seyedeh Hosna Talebian ${ }^{1}$ (D) Mohammad Enayati Najafabadi ${ }^{2} \cdot$ Ahmad Fahimifar $^{3}$
}

Received: 24 November 2020 / Accepted: 9 June 2021

Published online: 23 June 2021

(C) The Author(s) 2021 OPEN

\begin{abstract}
The focus of the present paper is on utilizing a comprehensive diagnostics workflow that combines coupled hydromechanical modeling with production data-driven diagnostics for optimization of stimulation candidate selection process. Reservoir fluids production and production-induced depletion affect reservoir mechanical environment due to reduced pore pressure and increased effective stress and the consequent porosity and permeability reduction, depending on rock sensitivity to stress changes. The coupled formation damage index (CFDI) is implemented in the traditional stimulation candidate selection workflow to capture the effect of production-induced stress changes on the near-wellbore permeability over time. The top potential stimulation candidates are recognized based on heterogeneity index, static formation damage index, and the CFDI parameter. CFDI is a dynamic parameter for stimulation candidate selection through estimating time-dependent permeability changes induced by stress state and fluid pressure. Probabilistic type curves and decline curve analysis of candidate wells versus reservoir unit are also applied to complement the stimulation and production enhancement candidate selection.
\end{abstract}

Keywords Data-driven stimulation candidate selection - Coupled hydro-mechanical model · Formation damage index · Heterogeneity index . Carbonate reservoir

\section{Introduction}

Carbonate reservoirs contain more than $50 \%$ of world's conventional hydrocarbon reserves and on average have relatively low recovery factors of $20-30 \%$, due to their inherent heterogeneity, complex pore system (matrix, fracture and vug), and wettability characteristics [1]. Due to a declining trend in the exploration of new super-giant fields and production decline of existing carbonate fields categorized as easy oil, better understanding of the productibility issues surrounding complex and tight carbonates such as permeability and fracture netwrok evolution has become increasingly important [2, 3]. Also in recent years, exploration and development companies have gained more interest in development of deep carbonates with more complexities in reservoir rock and fluid behavior and application of improved oil recovery (IOR) techniques such as stimulation to tackle productivity issues [4]. Uncertainties in microfracture network distribution and fluid behavior heterogeneities are some of the complexity elements that impose challenges to the IOR candidate selection and make the use of simulation models as the main way of data integration limited [5]. Therefore, the importance of surveillance data-driven analytics integrated with the

$\triangle$ Seyedeh Hosna Talebian, hsn.talebian@gmail.com | ${ }^{1}$ Oil, Gas, and Petroleum Engineering Research Center, Amirkabir University of Technology, Tehran, Iran. ${ }^{2}$ Department of Mining Engineering, Amirkabir University of Technology, Tehran, Iran. ${ }^{3}$ Department of Civil and Environmental Engineering, Amirkabir University of Technology, Tehran, Iran. 
hydro-mechanical modeling for the IOR decision-making process such as formation damage removal is highlighted [4]. The surveillance data-driven analytics integrated with the hydro-mechanical modeling to identify the stimulation candidate selection in a complex carbonate oil field is discussed in this paper.

In its basic definition, near-wellbore formation damage is any unintended impedance to the flow of fluids into or out of a wellbore, caused by a reduction in permeability in the near-wellbore region, the changes in relative permeability to the hydrocarbon phase and the unintended flow restrictions in the completion itself [6]. The flow restrictions in tubing or imposed by well completion geometry are not included in this definition. The sources of near-wellbore formation damage can be internal or external sources. Internal sources include organic or inorganic deposits from the produced fluids such as scales, asphaltene, and fines. External sources include emulsions, precipitates or sludges caused by acid reactions, clay swelling or wettability changes caused by injected fluids and entrained particles such as solids in injected fluids [7]. All downhole operations including drilling, cementing, perforating, completion, work-over, gravel packing, production/injection, and even stimulation process can induce the nearwellbore formation damage [8]. In some cases of acidizing, stimulation can cause damage due to use of incompatible fluids, not properly mixed fluids, sludge formation, water blocking, wettability alterations or post-treatment fines migration. A more general definition, however, considers the formation damage in the rock matrix caused by the changes to the chemical-biological-mechanical-thermal environment during processes of enhanced oil/gas and geothermal recovery [9]. The reservoir-based enhanced oil recovery (EOR) methods such as $\mathrm{CO}_{2}$ flooding, low salinity water (LSW), Alkali-surfactant-polymer (ASP) flooding, steam injection, and water injection can lead to reservoir formation damage.

One phenomenon that affects the mechanical environment of both conventional and unconventional reservoirs is the depletion due to production of oil and gas from the reservoir and consequently increased effective stress across the reservoir and compaction. The effective stress $\left(\sigma^{\prime}\right)$, which is defined as the difference between total stress $(\sigma)$, and pore pressure $(p)$, is the average normal force per unit area transmitted from grain to grain of the rock. Figure 1 shows the relationship between total stress, effective stress, and pore pressure in a rock buried deep into the reservoir and acted upon by the overburden stresses.

Depending on how sensitive is the reservoir to the stress changes, compaction reduces the porosity and permeability of the reservoir formation, with permeability to be a more stress-sensitive property than porosity. The compaction-induced permeability reduction hinders the

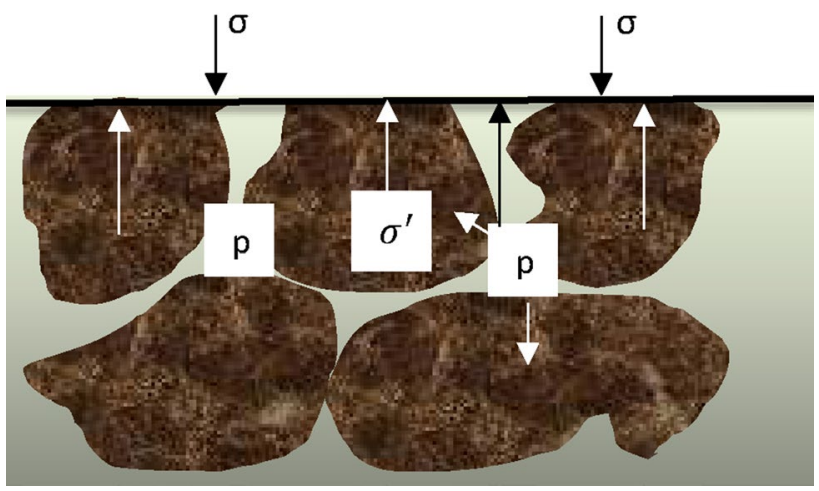

Fig. 1 Effective stress on rock grains in relationship with total stress and pore pressure [10]

production of the well and can act as a near-wellbore formation damage [10]. Although it is difficult to experimentally predict the depletion-induced stress changes due to scale effects, stress arching, and faulting contributions in the field, it was observed that most of stress changes during production are irrecoverable on repressurization [11]. This observation highlights the importance of depletion rate management to reduce irrecoverable formation damage.

Various permeability relations from field and laboratory experimental results have been suggested for carbonate reservoirs, starting with the work of Dake [12]. Some models derive permeability relations via correlating a big data of the porosity and the permeability pairs for worldwide carbonate reservoirs classified in terms of the reservoir location and the rock types [13]. However, there exist stress-based models [14], in which a power law is proposed for the variation of the permeability with the effective stress. Various conditions were applied on permeability relations [15], variable stress conditions [14], or triaxial strain/ stress conditions [16]. Some models were based on complex distribution features of reservoir types [17], or were derived for carbonate reservoirs with extremely limited logging data [18].

\subsection{Traditional stimulation candidate selection}

The candidate selection is the most important step of stimulation process, as applying the best treatment design and field procedures to the wrong candidate will result in failure [19]. According to the traditional stimulation candidate selection workflows, the candidates are selected through a process of production profile analysis, nodal analysis, perforation and completion review to determine damage mechanisms and logs to evaluate mineralogy [8]. The damage mechanisms that contribute to total pressure 
drop around the wellbore are considered as the components of total skin in well nodal analysis, as Eq. (1):

$S_{\text {total }}=S_{\text {dam }}+S_{c}+S_{p}+S_{\text {dev }}+S_{\text {stim }}+S_{\text {non-darcy }}+S_{\text {tp }}$

where $S_{\text {total }}$ is total skin, $S_{\text {dam }}$ is skin due to formation damage, $S_{c}$ is completion skin due to sand control or partial penetration, $S_{\mathrm{p}}$ is perforation skin due to crush zone and flow convergence, $S_{\text {dev }}$ is skin due to wellbore deviation that can be negative if perforated height is more than reservoir height, $S_{\text {stim }}$ is skin due to stimulation that can be zero by acid stimulation or negative for hydraulic fracturing, $S_{\text {non-darcy }}$ is rate-dependent skin that is prominent in gas well and $S_{\text {tp }}$ is skin due to two-phase flow, due to the competition of two flowing phases for the available flow channels [6]. The production-induced stress changes associated with depleting within reservoir and the consequent evolution of porosity, permeability and potential normal faulting are important to be studied in candidate selection process for further production enhancement and stimulation jobs. By using the coupled hydro-mechanical model that is described in the next section, one can estimate the total porosity loss in depleting reservoir and the associated permeability changes as a function of time.

\subsection{Coupling hydro-mechanical model (HM) approach}

In reservoir simulator approach, Darcy's law is solved for a case of single-phase, incompressible, isothermal flow, which in the absence of gravity can be written as Eq. (2).

$\phi \nu=-\frac{k}{\mu} \operatorname{grad} p$

with $\phi$ the porosity of the porous medium, $v$ the seepage velocity of the fluid in the porous medium, $k$ the rock permeability, $\mu$ the fluid dynamic viscosity, and $p$ the fluid pressure. In conventional simulation approach, rock permeability is assumed to be constant or depend only on fluid with uniaxial or isotropic strain for each mesh of the reservoir. In actual reservoir conditions, pressure, temperature and saturation variations generated by exploitation modify the stress distribution in the reservoir. Consequently, the porosity and compressibility of the rock vary during the reservoir production. According to Gutierrex and Lewis [20], the pressure dependence of porosity imposed via the rock compressibility introduced in reservoir simulators cannot alone describe the behavior of the reservoir rock during production and depends on the stress path followed by reservoir during its depletion. The stress path is the change in stress due to the change in pore pressure at a given point in the reservoir. It is defined as $\Delta \sigma_{\mathrm{h}} / \Delta p$ where $\Delta \sigma_{\mathrm{h}}$ is the change in horizontal stress and $\Delta p$ is the change in pore pressure. If the depleting reservoir is infinite, i.e., the lateral length exceeds 10 times the reservoir thickness, using instantaneous application of force and pressure with no lateral strain, yields to a simple equation [Eq. (3)] proposed to minimum horizontal stress in an isotropic basin [21]:

$\sigma_{\mathrm{h}}=\left(\frac{v}{1-v}\right) \sigma_{\mathrm{v}}+b P_{\mathrm{p}}\left(1-\frac{v}{1-v}\right)$

where $\sigma_{\mathrm{h}}$ is minimum horizontal stress, $v$ is Poisson's ratio, $\sigma_{\mathrm{v}}$ is overburden stress, $b$ is Biot's coefficient and $P_{\mathrm{p}}$ is the pore pressure [22]. Taking the derivative of both sides of Eq. (3), and after simplification:

$\Delta \sigma_{\mathrm{h}}=b\left(\frac{1-2 v}{1-v}\right) \Delta P_{\mathrm{p}}$

In Eq. (4), it is assumed that overburden stress is not coupled with pore pressure, and therefore, the overburden stress vanishes during the derivation of Eq. (3) and $\Delta \sigma_{v}=0$. Such an assumption was systematically studied in the way that minimum horizontal stress is fully coupled with pore pressure and is calibrated with hydraulic fracture stress data [23]. However, as researchers measured the effect of reservoir geometry and boundaries on the stress path [20,24], the necessity of using an empirically determined effective Poisson's ration which is calibrated against the least principal stress measurements obtained from leak-off tests is recognized. Therefore, the use of laboratory-defined Poisson's ratio for a reservoir in Eq. (4) is based on strong assumptions of uniaxial reservoir compaction, elastic behavior of the rock during depletion and homogenous drained Poisson's ratio at reservoir scale, identified to that measured in laboratory scale [10].

Poroelastic theory is applied in oil and gas industry mainly to understand subsidence, estimate reservoir productivity, and predict stresses around wellbores [25]. The carbonate reservoir is considered to be homogeneous. The elastic behavior of the reservoir is considered to be linear and isotropic elastic behavior. Only small strains are considered. The pore space is saturated with oil. Molecules in the pore space are assumed to be in a bulk state. Uniaxial strain behavior is considered for boundary conditions [11]. We consider a nonporous matrix in a representative elementary volume (REV) of the carbonate reservoir, i.e., the carbonate reservoir could be considered as a regular porous medium made of one pore network (i.e., the network of fractures). Therefore, the energy balance for the nonporous matrix in a REV of carbonate reservoir would be as Eq. (5) [26]:

$d f=\sigma \mathrm{d} \epsilon+s_{i j} \mathrm{~d} e_{i j}+p \mathrm{~d} \phi$

where $f$ is defined as the Helmholtz free energy of the matrix per unit volume of reservoir. $\sigma, s_{i j}$ and $p$ are the 
volumetric stress, the deviatoric stresses, and the pore pressure, respectively. $\epsilon, e_{i j}$, and $\phi$ are the volumetric strain, the deviatoric strains, and the Lagrangian porosity, respectively. Energy can be added to such a system either by straining it with volumetric confining stress $\sigma$ (the corresponding work being ' $\left.\sigma \mathrm{d} \varepsilon^{\prime}\right)$, by straining it with a deviatoric confining stress $s_{i j}$ (the corresponding work being ' $s_{i j} \mathrm{de} e_{i j}{ }^{\prime}$ ) and by deforming the porosity $\phi$ with the pressure of the fluid in the matrix, $p$ (the corresponding work being ' $p d \phi^{\prime}$ '). Based on this energy balance, one can write the constitutive equations of the carbonate reservoir as [26]:

$$
\begin{aligned}
& \mathrm{d} \sigma=\left(K+b^{2} N\right) \mathrm{d} \epsilon-b N \mathrm{~d} \phi \\
& \mathrm{d} p=-b N \mathrm{~d} \epsilon+N \mathrm{~d} \phi \\
& \mathrm{d} s_{i j}=2 G \mathrm{~d} e_{i j}
\end{aligned}
$$

where $K, b, N$, and $G$ are poroleastic constants defined as the drained compression modulus, the Biot's coefficient, the Biot's modulus, and the shear modulus, respectively, all associated to the reservoir. Based on the derived constitutive equations, Eqs. (2)-(4), we performed carbonate reservoir simulation. The performed simulations were asymmetric plane-strain two-dimensional. A Kozeny-Carman-type equation for permeability in the reservoir is considered, in which only the porosity intervened. Hence, the permeability $k$ is given via the following relation [27]:

$\frac{k}{k_{0}}=\frac{\phi^{3}}{(1-\phi)^{2}} \times \frac{\left(1-\phi_{0}\right)^{2}}{\phi_{0}^{3}}$

where $k_{0}$ and $\phi_{0}$ are the permeability and porosity in the state of reference and $\phi$ is the porosity at the actual state. Equation (9) shows that permeability ( $k$ ) varies non-trivially with the porosity; with increase of permeability, porosity increases.

The derivations performed in Eqs. (5-7 and 9) help to understand and predict how parameters such as porosity or permeability change for a representative elementary volume of the case carbonate reservoir saturated by oil. Moreover, a significant feature of our model is that twoway coupling between pore pressures and stresses or strains is captured: not only does the two-way behavior make it possible to predict how stresses or strains affect pore pressure, but also does it make it possible to predict how stresses or strains change in the presence of pore pressure. In other words, our model is able to capture the effect of stresses or strains on the permeability of the reservoir over time.

\section{Applied stimulation candidate selection workflow}

The proposed workflow in the current study is presented in Fig. 2, to screen the potential candidate wells for stimulation within a particular producing carbonate reservoir.

The presented diagnostics workflow is based on the monitored production data-driven analytics, well-test data acquired at the first day of production and coupled hydromechanical simulation accounting stress changes around the wellbore area. Figure 3 represents the schematic diagram of the established 2D coupled finite element model that used for investigating the effect of near-wellbore stress changes at perforation depth of $3500-4000(\mathrm{~m})$ for all the wells in the producing reservoir layer.

As described in Fig. 2, the heterogeneity index $(\mathrm{HI})$ is a quick screening process to identify the preliminary candidate wells with anomalous behavior by comparing the relative production performance of each individual well to the performance of the field average [28]. The crosshair $\mathrm{HI}$ plot provides a view of individual well performances as better or worse than the field average in four quadrants. According to this plot, the candidates with oil and water production history lower than the average field performance proceed to the stimulation candidates' pool. The candidates with water production higher than the average field performance and oil production lower than the average field performance proceed to water shut-off (WSO) candidate selection workflow. Candidates with water cut higher than $50 \%$ may cause risk to the stimulation gain. Finally, the candidates with oil and water production history higher than the average field performance according to this plot are followed by continuous well performance monitoring. The formation damage indicator (FDI) plot is a scatter plot of reservoir capacity vs. the maximum oil rate for each well. The reservoir capacity is defined a permeability of the perforation zone obtained during well test multiplies the perforation interval $\left(k_{0} h\right)$. This scatter plot is to detect the underperforming well possibly due to skin, even though the formation has a high storage capacity to deliver. Thus, the reserves may remain trapped in a high percentage of the potentially productive zone. Considering the $k_{0} h$ values are not changing with time, it's a static plot.

Coupled formation damage index plot (CFDI) addresses the permeability ( $k$ ) from Eq. (9) which is governed by porosity coupled with stresses or strains [Eqs. (5-8)]. Thus, CFDI plot is a dynamic scatter plot that takes into account the stress changes during the reservoir production and the associated permeability changes of each well during its production time. 


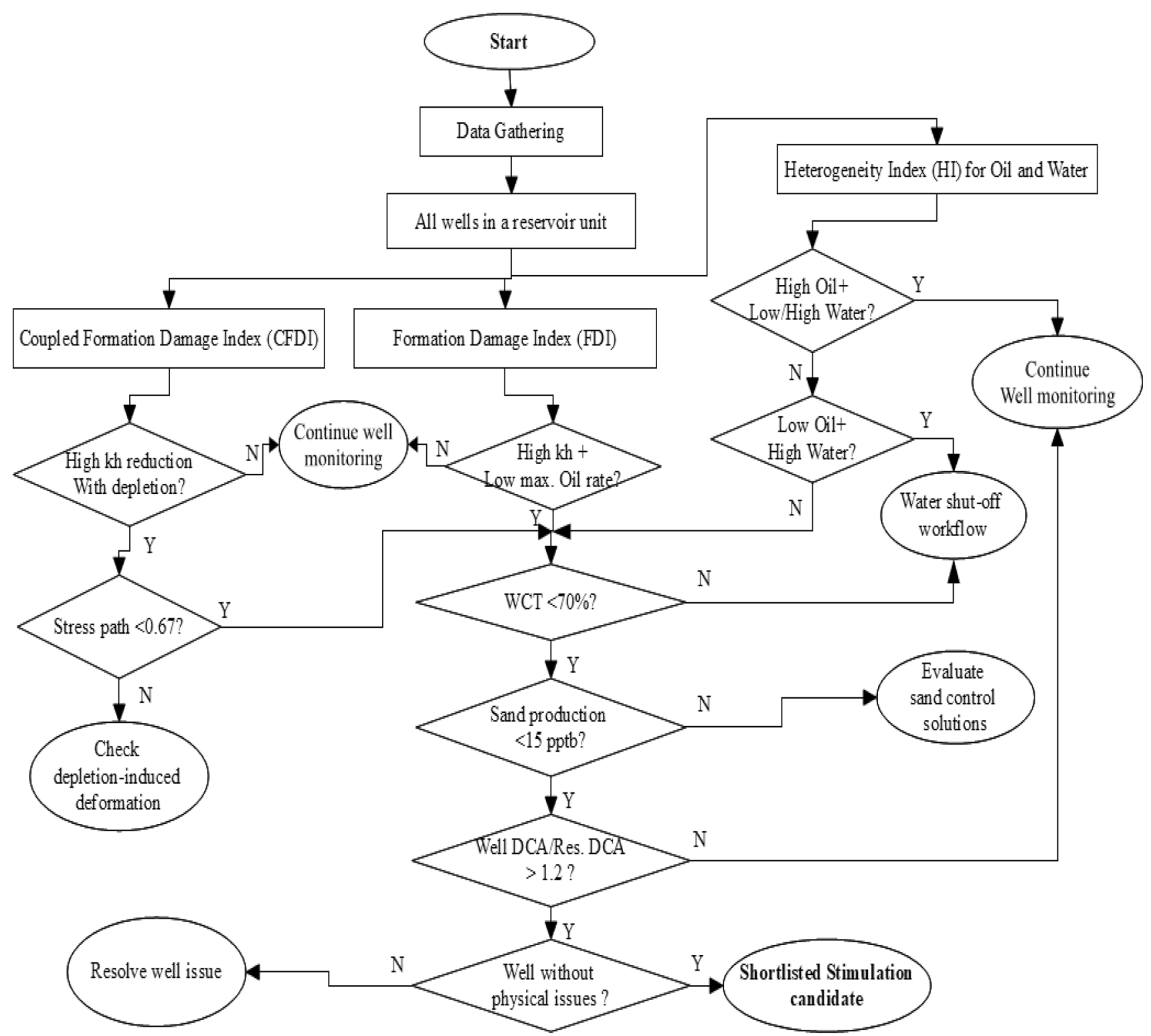

Fig. 2 Proposed candidate selection workflow

According to the procedure developed by Zoback [10], by using Eq. (4) and setting $b=1$ and $v=0.25$, one can find stress path as $\Delta \sigma_{\mathrm{h}} / \Delta p=0.67$. When the stress path is above 0.67 , the production can induce normal faulting and the depletion-induced deformation should be checked. However, when the stress path is below 0.67 , it would be a stable stress path.

Once the candidates have been screened based on $\mathrm{HI}$ plot, FDI and CFDI plots, the wells are analyzed individually to investigate the current status of the well and reservoir properties. Production performance of individual wells needs to be monitored to eliminate the inappropriate candidates such as the ones with high water cut percentage, i.e., above $70 \%$ [29]. If the produced sand concentration in a candidate well is higher than the field critical value, i.e., $15 \mathrm{lb}$ per thousand barrels (pptb) [30], stimulation can hinder erosion risk and sand control options are the priority for the well, especially in gas wells. As the stimulation candidates are highly recommended if the reserves are sufficiently high enough to satisfy the fast payout time, the well decline curve is compared against the reservoir decline curve as the next step of screening process. If the reservoir pressure is available, average reservoir pressure decline is checked to be less than $5 \%$ to ensure the well decline is not due to the reservoir pressure decline. Before shortlisted as stimulation candidate, production performance for each candidate has to be investigated for the events such as shut-in well due to mechanical issue, 


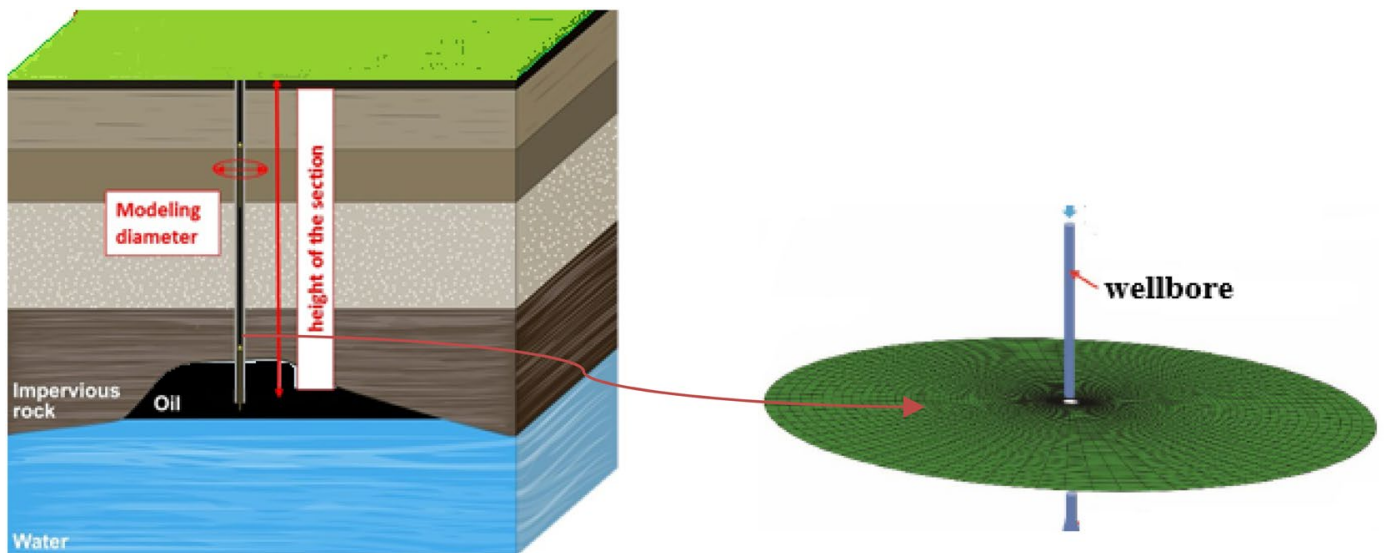

Fig. 3 Schematic of wellbore trajectory and model geometry for hydro-mechanical model

Table 1 Poromechanical parameters for the case carbonate field studied

\begin{tabular}{lll}
\hline Parameter & Definition & Value \\
\hline$E$ & Young modulus of reservoir (psi) & $5,000,000$ \\
$P_{\mathrm{P}}$ & Initial liquid pore pressure (psi) & 7250 \\
$v$ & Poisson's ratio of reservoir & 0.30 \\
$K$ & Bulk modulus of reservoir (psi) & $4,000,000$ \\
$b$ & Biot coefficient of reservoir & 1.00 \\
$\phi_{0}$ & Initial porosity (\%) & Reported in \\
$k_{0}$ & Initial permeability (mD) & Table 2 for \\
Sp. Gr & Specific gravity oil (API ${ }^{0}$ each well & 32.00 \\
\hline
\end{tabular}

Table 2 Well characteristics at the reservoir unit cross section

\begin{tabular}{lcc}
\hline Well no & $k_{0} h(\mathrm{mD} . \mathrm{ft})$ & $\phi_{0}(\%)$ \\
\hline 003 & 1559.95 & 7.00 \\
005 & 1679.93 & 7.00 \\
006 & 3939.88 & 6.00 \\
007 & 1575.96 & 8.00 \\
009 & $23,621.3$ & 8.00 \\
011 & 1575.94 & 5.00 \\
012 & 4068.13 & 8.00 \\
013 & 1541.96 & 8.00 \\
\hline
\end{tabular}

abandon well or zone change that makes it inappropriate for the stimulation.

\section{Field application and analysis}

Parameters used for REV of the case carbonate reservoir are listed in Tables 1 and 2. A finite element model is used to perform the two-dimensional coupled hydro-mechanical model for a radius of $50 \mathrm{~m}$ of matrix rock around wellbore area at the mid-perforation depth of each individual well with a number of $40^{\prime} 000$ grid blocks. A sensitivity analysis was performed on number of grid blocks from 1500 to $42^{\prime} 000$, and as changes in results approached zero by addition of grid blocks above 40,000 , the mesh-size of 40,000 for our modeling was selected. The Biot coefficient (b) of the reservoir is required to be assumed in the simulations. Using stress path criteria explained in previous section and by setting Biot coefficient $b=1$ and Poisson's ratio $v=0.3$ from Table 1, the stress path would be 0.57 . Since the stress path in this study (i.e., 0.57 ) is shallower that 0.67 , the stress and pore pressure will evolve with flatter gradient and get further from the failure condition (i.e., 0.67), thus the depletion is stabilizing the reservoir and the faulting would not happen.

The designed workflow as per Fig. 2 has been utilized in a complex carbonate field with microfracture network system for stimulation candidate selection process. A continuous hydro-mechanical modeling approach was selected for this case due to uncertainties in microfracture network that implies single-medium porous system. The single completion production wells penetrate multiple formation layers vertically, where only one continuous shale barrier separates the two main producing layers. The parameters for $\mathrm{HI}$ plot of oil and water production performance in $\mathrm{y}$ and $x$ axis are presented in Eqs. (10) and (11), respectively.

HISUMW $=\frac{Q_{w} \text { well }}{Q_{w} \text { average of wells }}-1$

HISUMO $=\frac{Q_{0} \text { well }}{Q_{0} \text { average of wells }}-1$

Based on Eqs. (10) and (11), the production wells that fall into negative HISUMO and HISUMW regions during their production history, which in this case are W003 and

\section{SN Applied Sciences}


W011, are selected as the first candidate group in stimulation candidate pool. As can be seen in Fig. 4, W003 has been underperforming throughout its production history, while W011 has just recently been added in the negative region in terms of oil and water production.

Figure $5 \mathrm{a}, \mathrm{b}$ are presenting the scatter plot of $k_{0} h$ versus maximum oil rate of wells and $k h$ map of wells across the reservoir unit of the studied case, respectively. As seen in Fig. 5a, although W012 has a high reservoir capacity based on the $k_{0} h$ value extracted from the initial welltest report, the maximum oil rate the well is comparatively lower than the other wells with similar formation capacity. Also as presented in Fig. $5 \mathrm{~b}$, the $k_{0} h$ values of well across the reservoir unit are categorized in three groups in terms of values. Apart from W009 that has shown a very high formation capacity at the initial welltest due to the microfracture network effect around the wellbore, the rests of the wells have almost same values with W012 and W006 showing
Fig. $4 \mathrm{HI}$ plot of the case study field
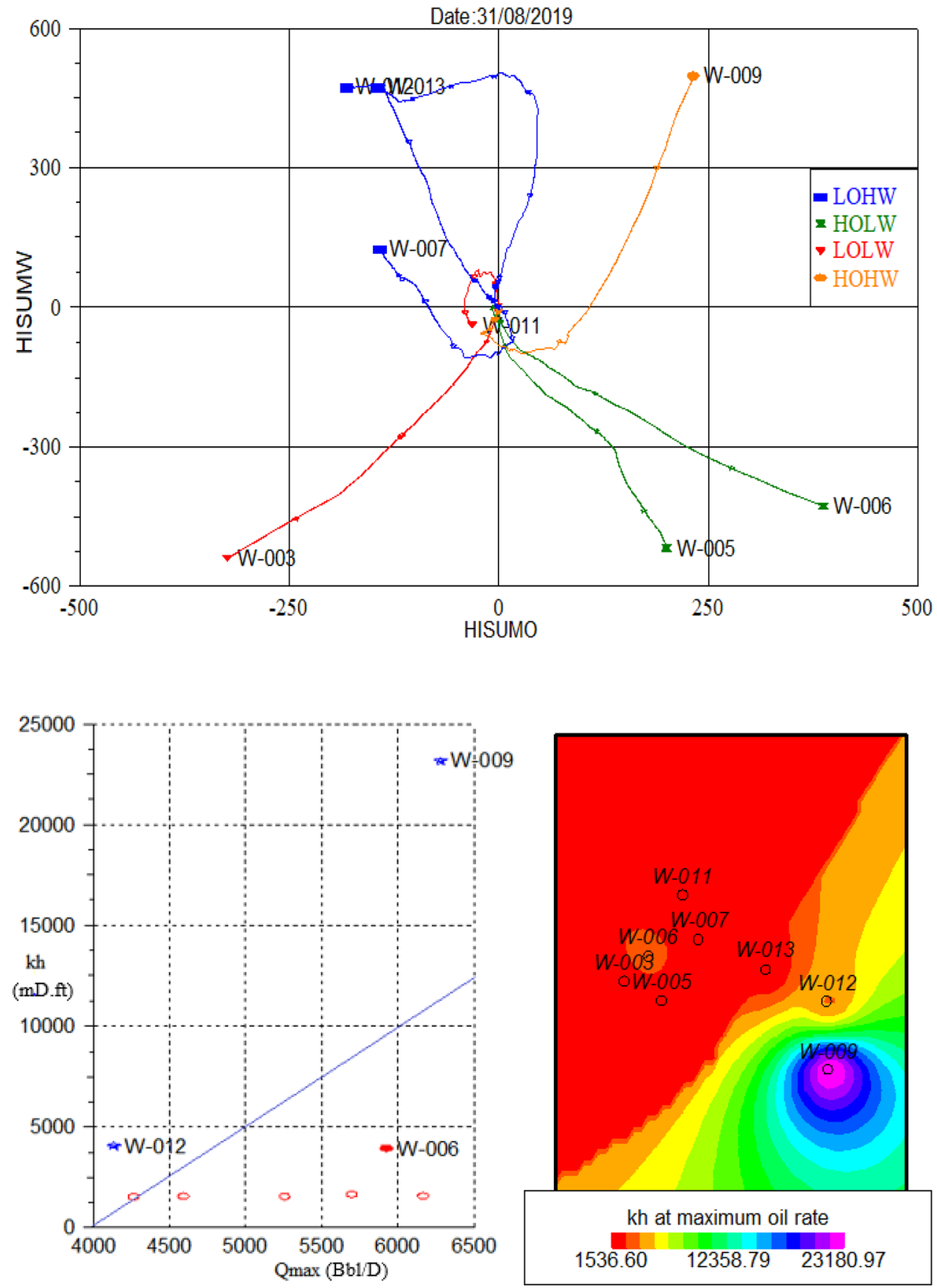

(a)

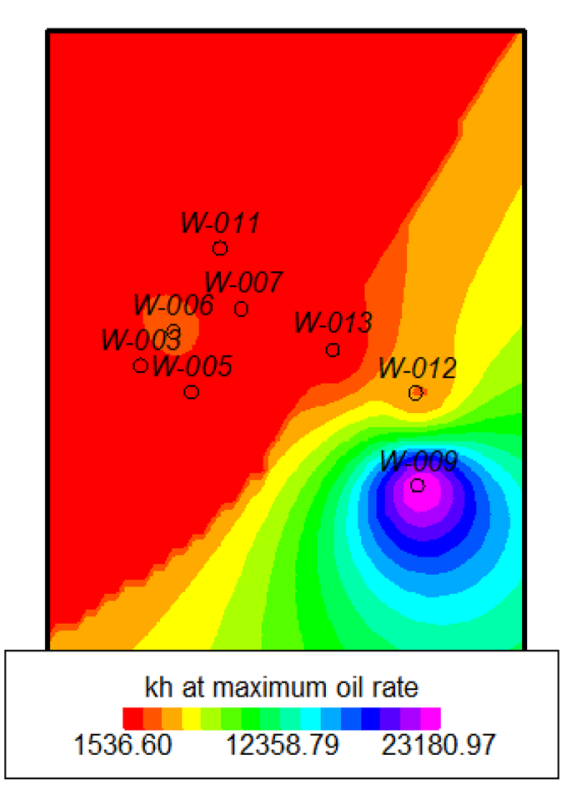

(b) 
relatively higher capacity. However, the maximum oil rate production monitored in W012 is so much lower than the one observed in W006, which makes W012 a potential stimulation candidate to carry on to the candidate pool.

Figures $6 \mathrm{a}-\mathrm{d}$ are presenting the results of coupled HM model for the changes in $k h$ and oil rate versus time for the four categories of wells as defined in $\mathrm{HI}$ plot. As can be seen in Fig. 6a, the changes in kh values for W011 is more highlighted than W003 in this group of wells as low productive wells. For the group of wells in low oil and highwater production as shown in Fig. 6b, W012 has presented higher kh changes during its production history. For the
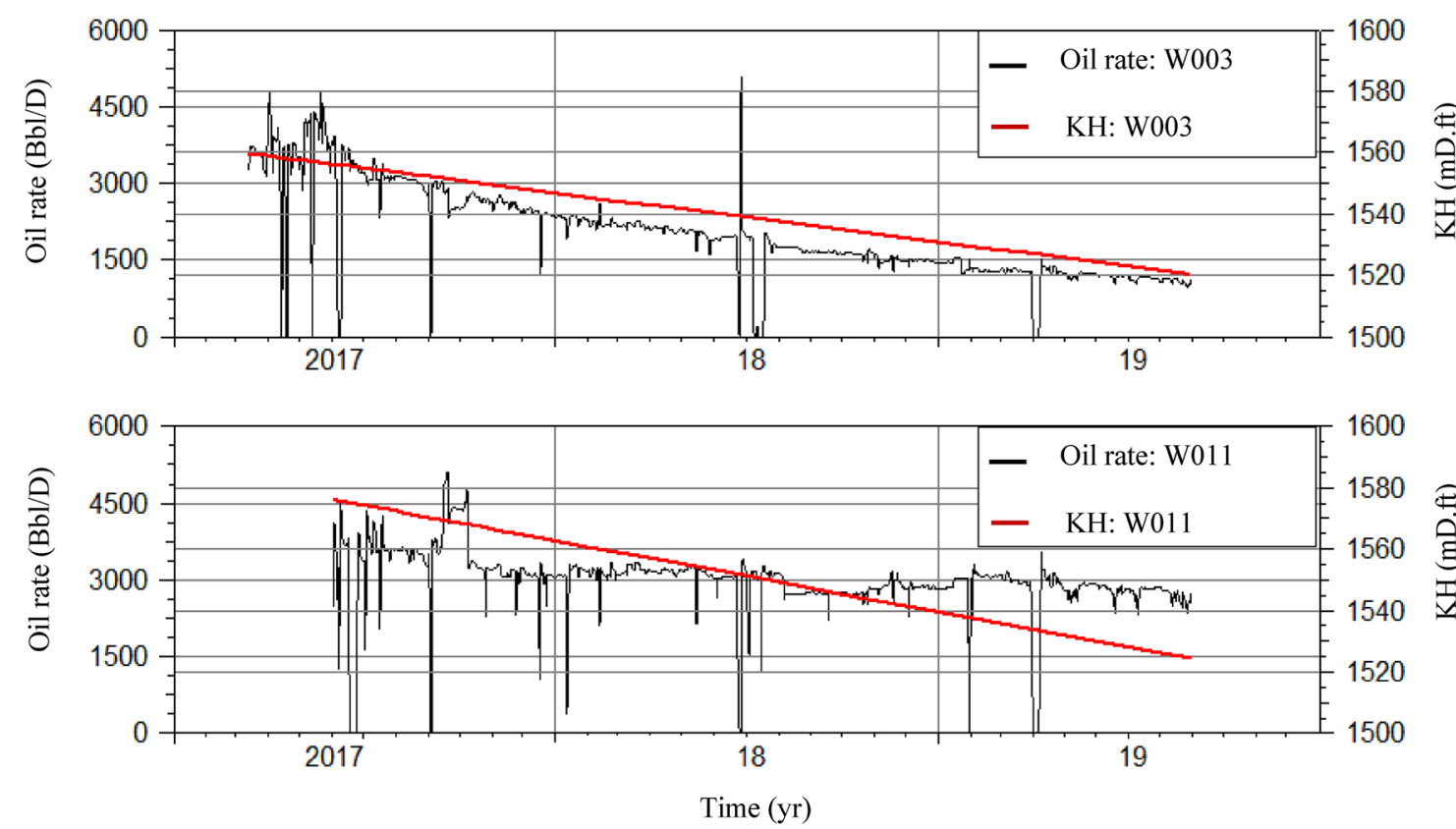

(a)
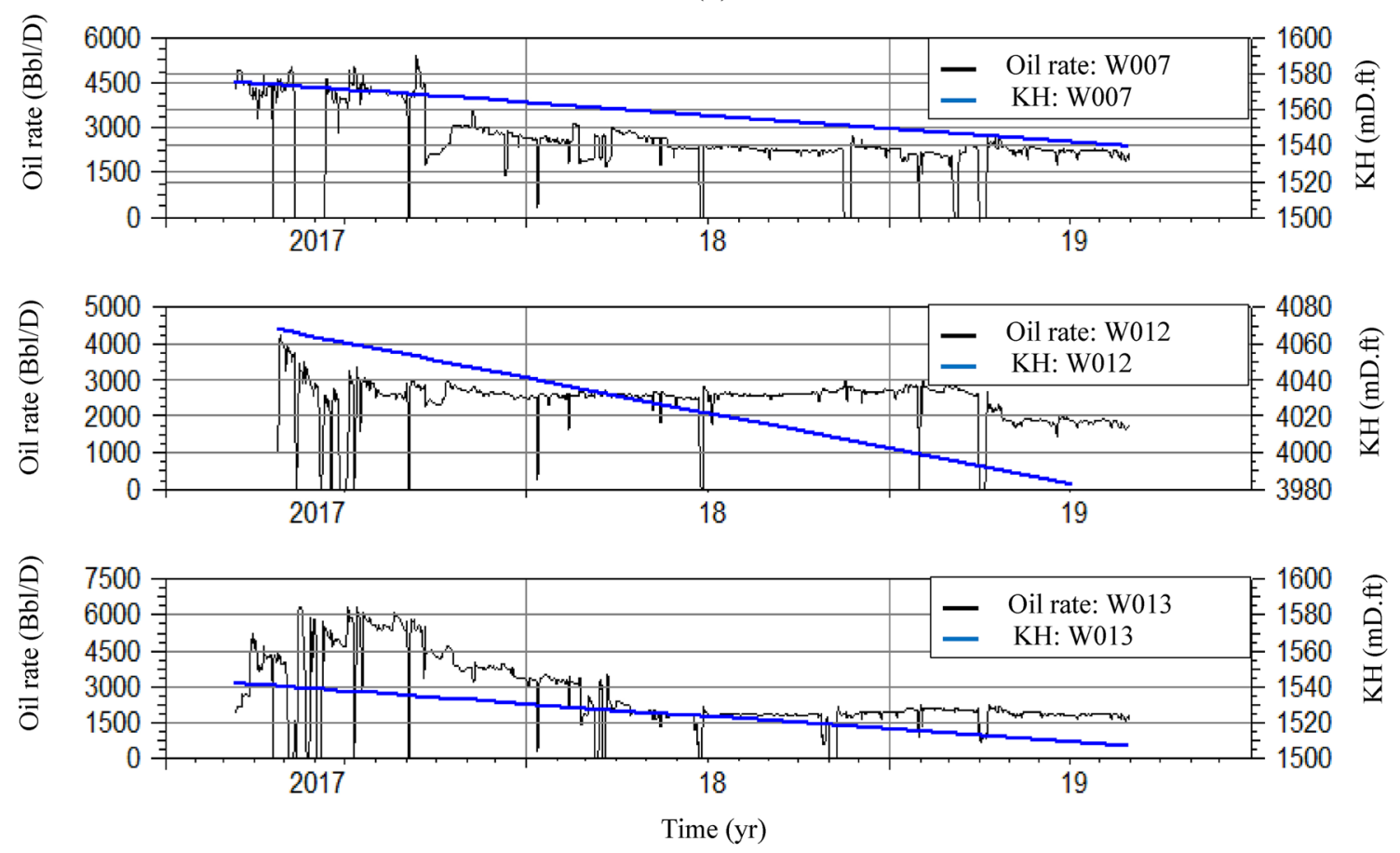

志

(b)

Fig. $6 \mathrm{kh}$ and oil rate vs. time for a low productive wells, $\mathbf{b}$ wells with high water and low oil production, $\mathbf{c}$ high oil and water production 


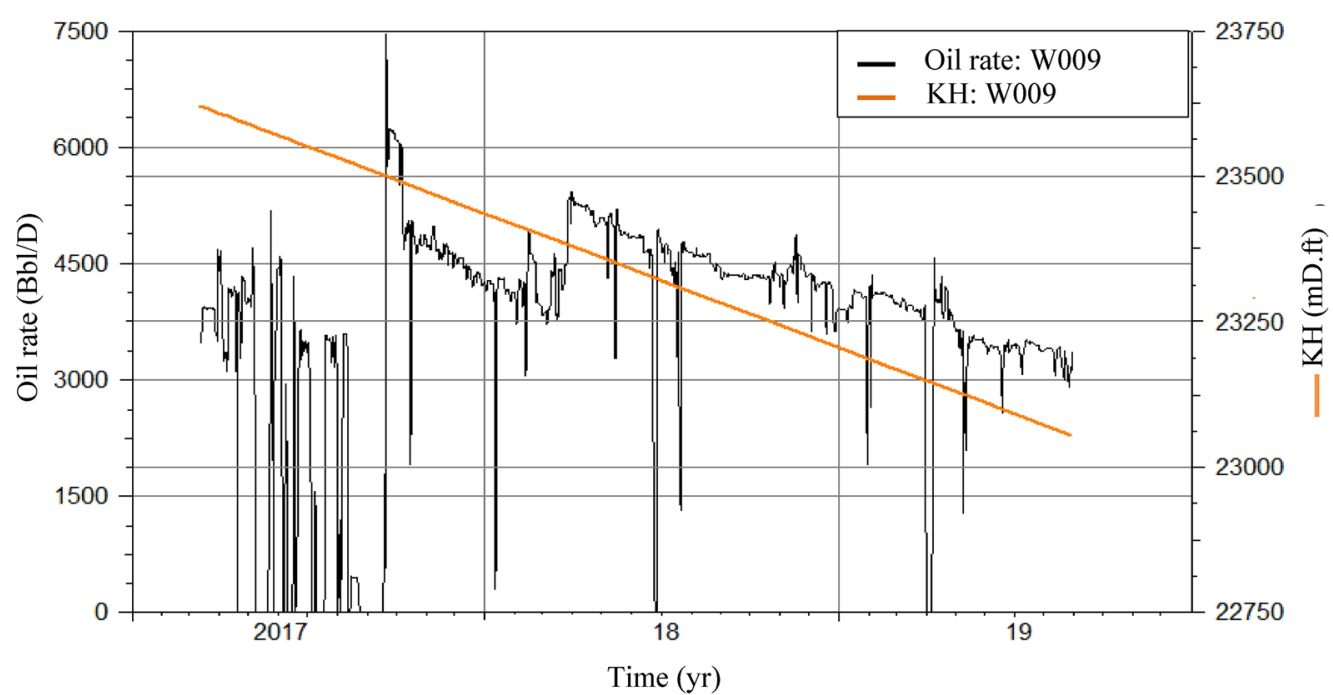

(c)
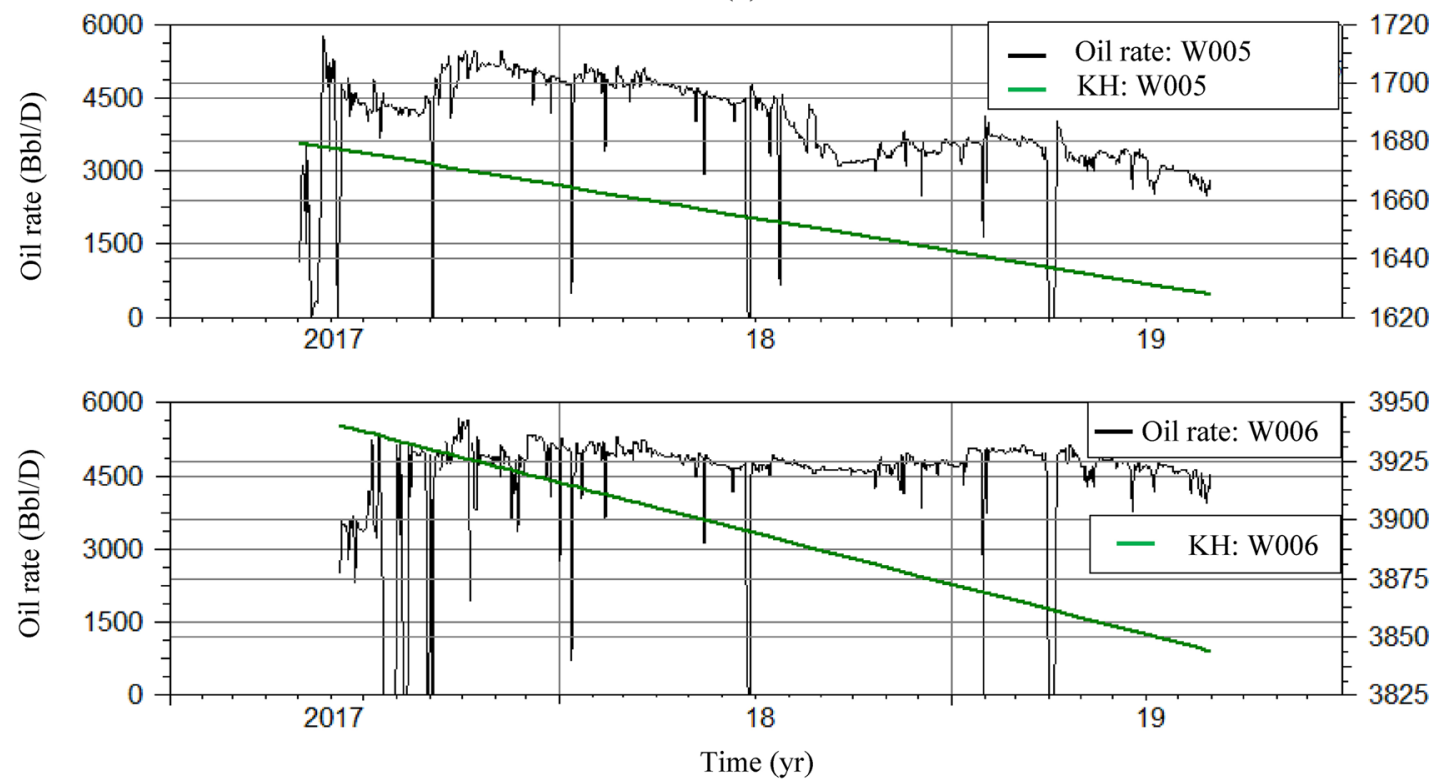

है
है
国

(d)

Fig. 6 (continued)

high productive group with high oil and high water production as seen in Fig. 6c, the kh change in W009 is nearly 1000 (mD.ft), which is high in three years of production with 3000 psi reduction in pressure. The comparison of $k h$ changes in the high productive group wells with high oil production and low water production, defines changes in W005 is more highlighted than in W006, which makes W005 also a candidate in the stimulation pool.

Considering that according to Fig. $6 \mathrm{~b}$, all the wells are in the same range of $k h$, except W006, W009 and W012, the $k h$ changes over time are plotted in Fig. 7 against the production time.
The grid maps of initial porosities and the flowing wellhead pressure of wells at the target reservoir unit are presented in Fig. 8a, b.

The selected candidates at this step are considered to proceed to DCA analysis and comparison of each candidate well's DCA against the reservoir unit DCA to ascertain the reservoir depletion is not the reason for the well's underperforming. The DCA plots of each candidate are shown in Figs. 9, 10 and 11. The DCA plot of the target reservoir unit is illustrated in Fig. 12.

The candidate wells from the same reservoir are compared with the average reservoir decline to provide another indication of production impairment. Table 3 
Fig. 7 kh changes over production time for the wells with the same range of initial $k_{0} h$
Fig. 8 a Grid map of wells initial porosities, $\mathbf{b}$ grid map of wellhead pressure at a certain time

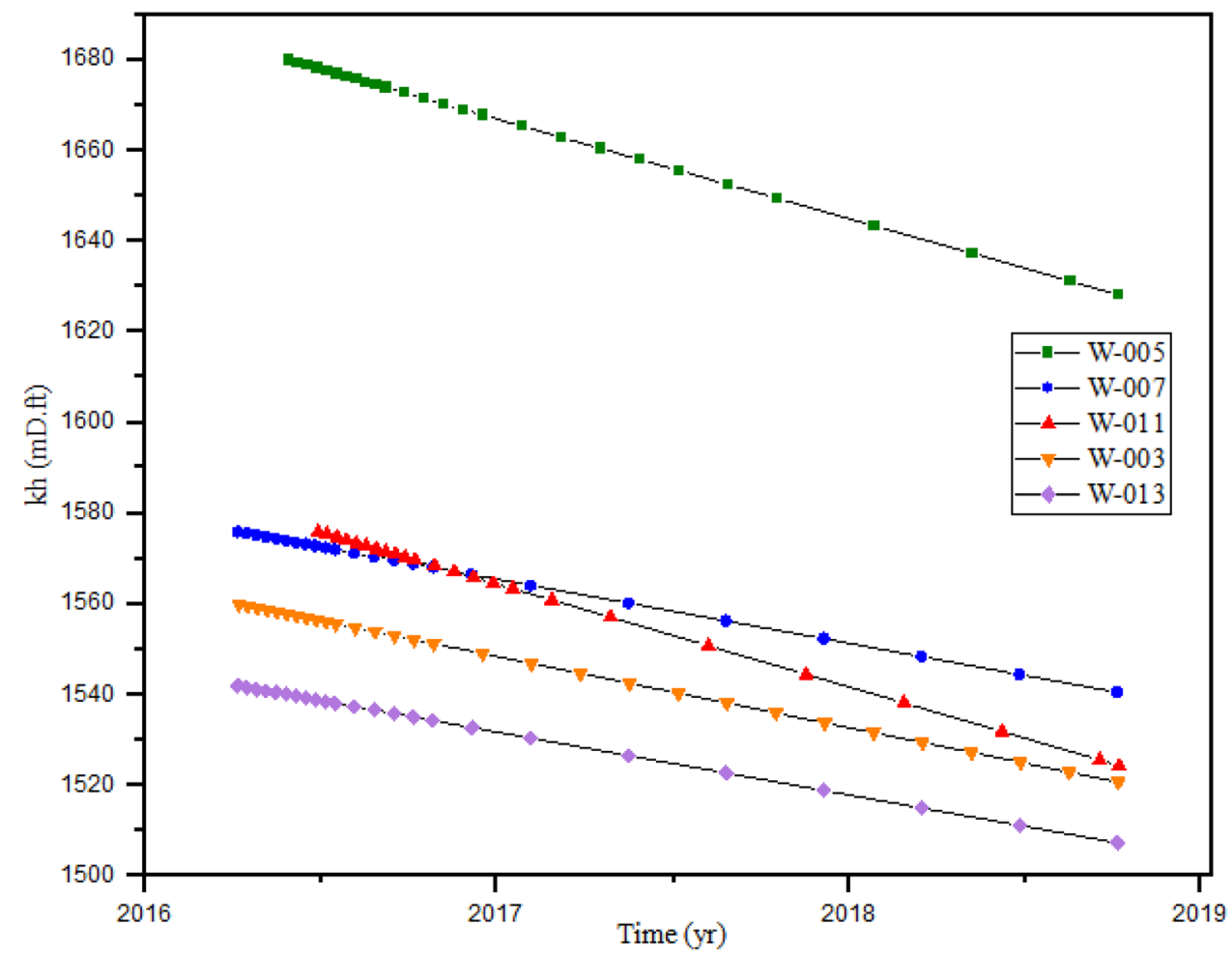

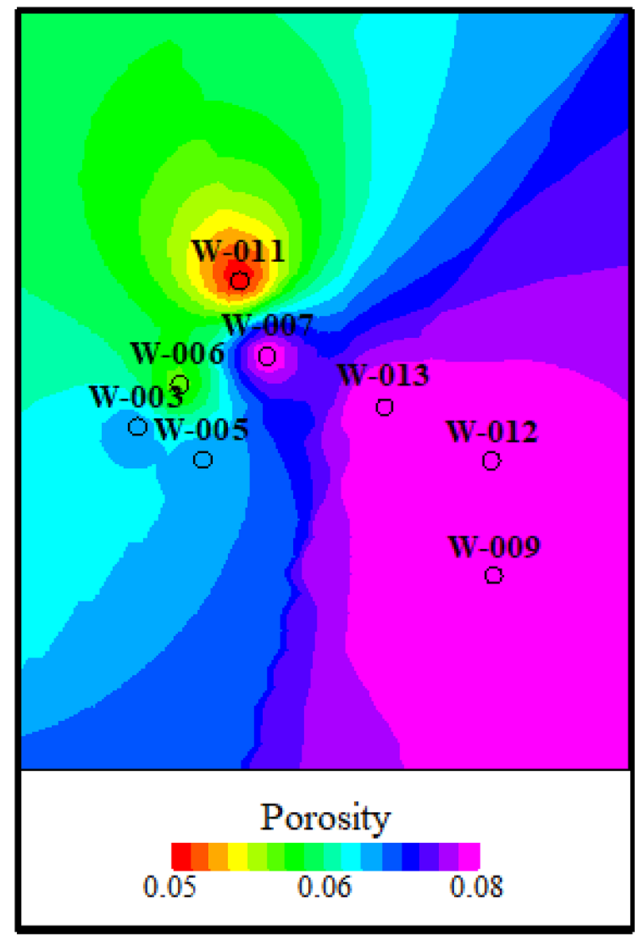

(a)

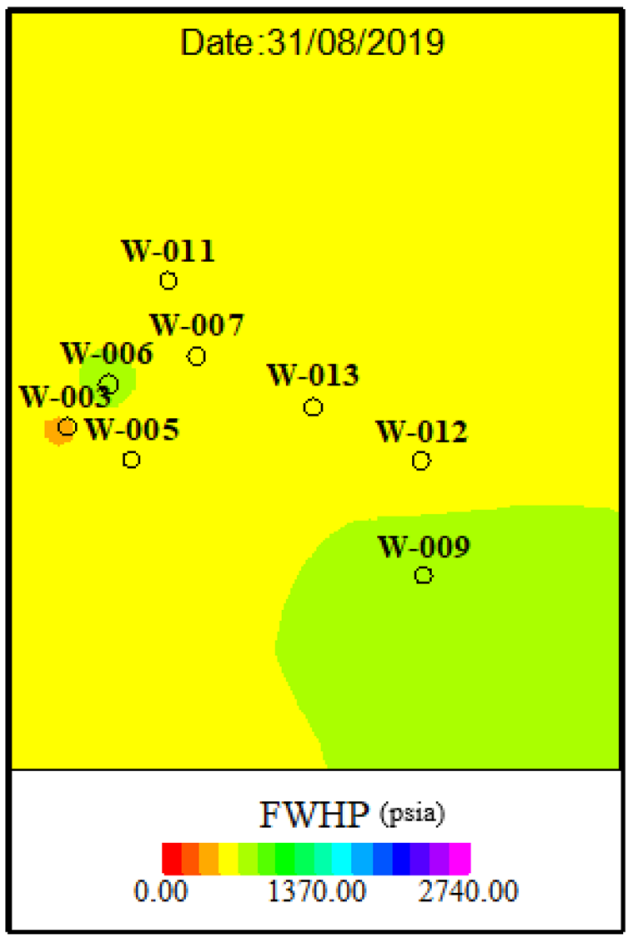

(b) reports the decline ratio of the well to the reservoir for all three candidates, which is above 1.2 for all three candidates. Therefore, the stimulation candidate wells are recommended as the reserves are sufficiently high enough to ensure the job is economic. 
Fig. 9 Rate versus time DCA for candidate W005
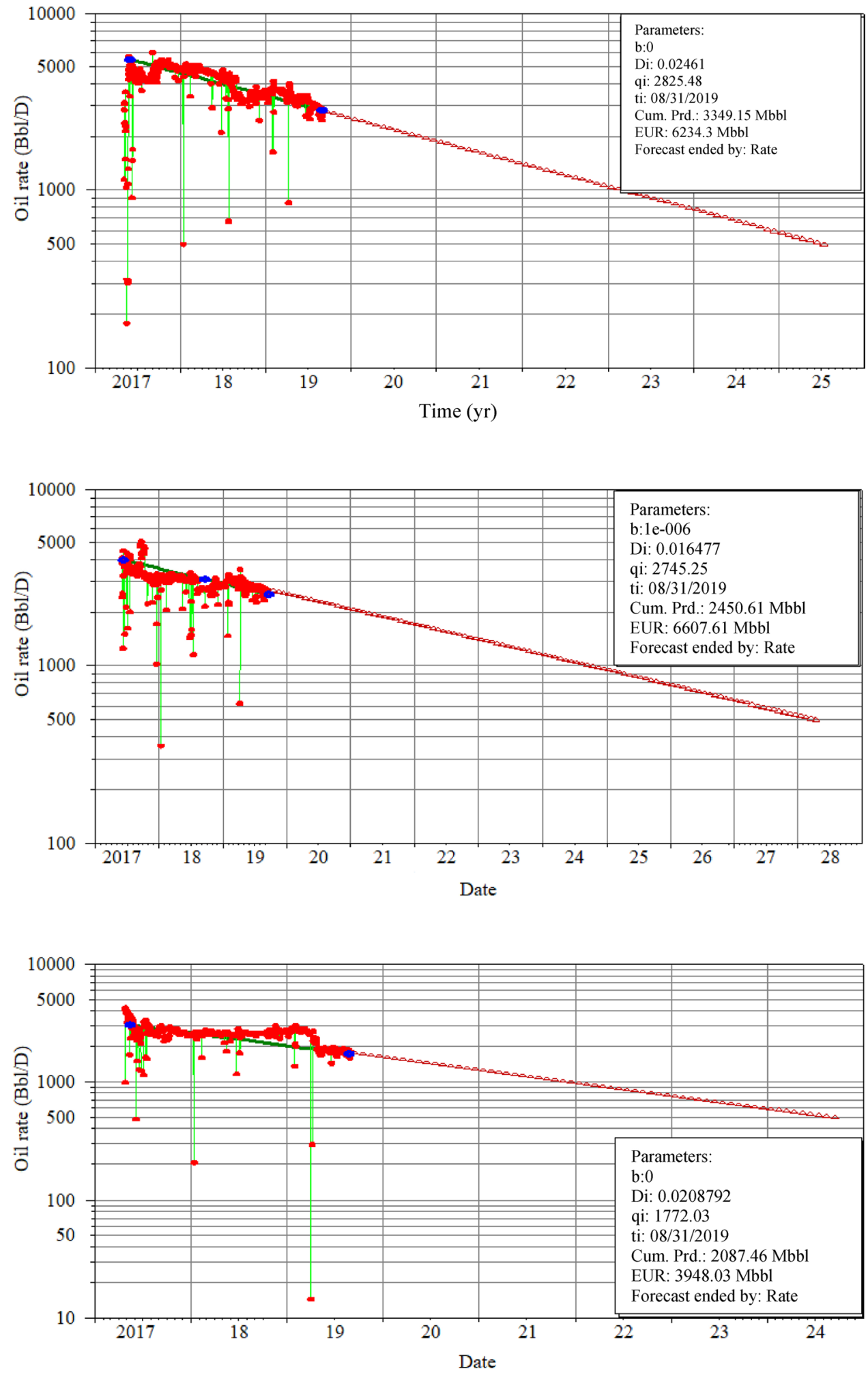

Fig. 11 Decline curve analysis (DCA) for candidate W012
Fig. 10 Rate versus time DCA for candidate W011

\section{Conclusions}

A diagnostics workflow for stimulation candidate selection based on production data-driven analytics integrated with the coupled hydro-mechanical model has been successfully developed and implemented in a complex carbonate field case. The proposed screening workflow takes into account the dynamic behavior of formation damage index over the production history 
Fig. 12 Decline curve analysis (DCA) for target reservoir unit

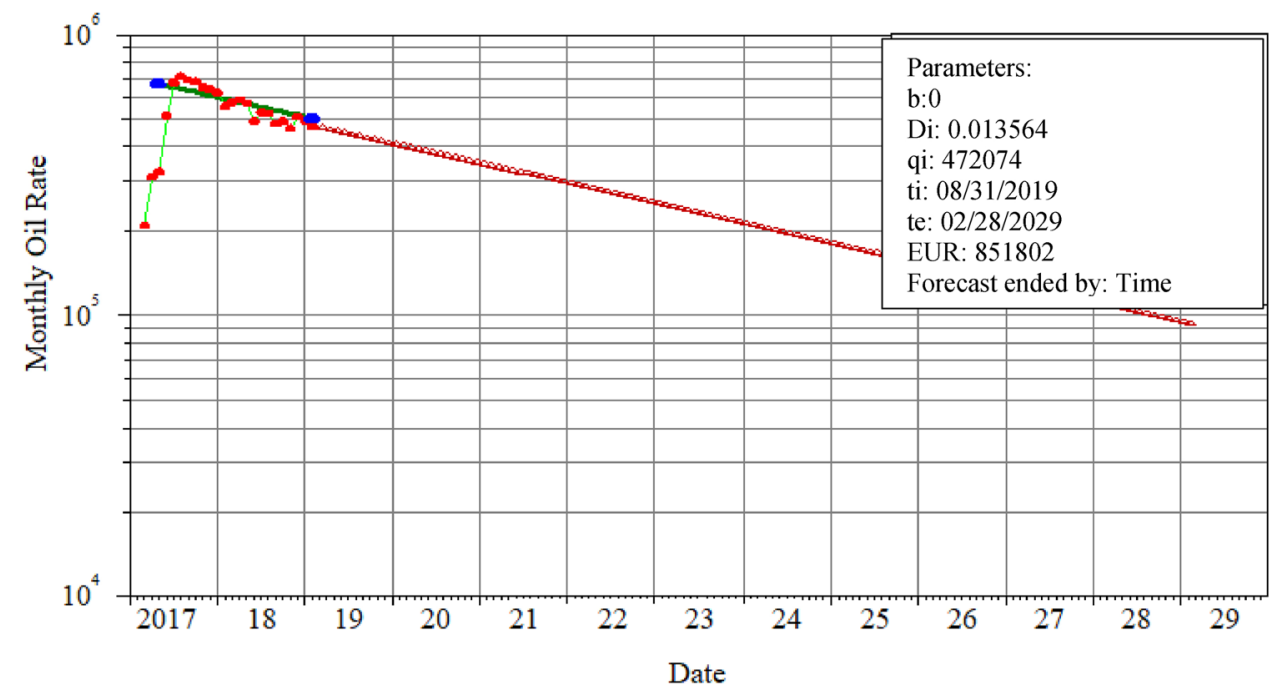

Table 3 Decline ration of candidate well to target reservoir

\begin{tabular}{ll}
\hline Well no & $\begin{array}{l}\text { DCA well/ } \\
\text { DCA reser- } \\
\text { voir }\end{array}$ \\
\hline 005 & 1.92 \\
011 & 1.23 \\
012 & 1.61 \\
\hline
\end{tabular}

Open Access This article is licensed under a Creative Commons Attribution 4.0 International License, which permits use, sharing, adaptation, distribution and reproduction in any medium or format, as long as you give appropriate credit to the original author(s) and the source, provide a link to the Creative Commons licence, and indicate if changes were made. The images or other third party material in this article are included in the article's Creative Commons licence, unless indicated otherwise in a credit line to the material. If material is not included in the article's Creative Commons licence and your intended use is not permitted by statutory regulation or exceeds the permitted use, you will need to obtain permission directly from the copyright holder. To view a copy of this licence, visit http://creativecommons. org/licenses/by/4.0/. of each well, by utilizing the coupled hydro-mechanical model to enhance the screening process and improve opportunity detection for stimulation jobs. The novelty of the applied workflow is that wells which are experiencing severe changes in near-wellbore permeability during their production history are detected to be considered for stimulation jobs, along with underperforming wells in comparison to the filed average. The integration of dynamic parameters such as flowing wellhead pressure and static parameters such as porosity, through the coupled hydro-dynamic model, provides a more comprehensive and enhanced stimulation candidate selection for the complex carbonate field of study. Another outcome of the approach is the automaton of data diagnostics process for saving significant manhours for the engineers and equipping managers with smart decision-making tool.

\section{Declarations}

Conflict of interest The authors declare that they have no conflict of interest.

\section{SN Applied Sciences}

\section{References}

1. Al-Shalabi EW, Sepehrnoori K, Delshad M (2014) Optimization of the low salinity water injection process in carbonate reservoirs. In: International petroleum technology conference. International Petroleum Technology Conference, Kuala Lumpur, Malaysia, p 28

2. Masalmeh SK et al (2014) EOR options for heterogeneous carbonate reservoirs currently under waterflooding. In: Abu Dhabi international petroleum exhibition and conference. Society of Petroleum Engineers, Abu Dhabi, UAE, p 29

3. Pattnaik C, et al (2015) Field development and well planning in tight carbonate reservoir using fracture characterization and in-situ stress mapping from core reorientation studies: Kuwait case study. In: SPE Kuwait oil and gas show and conference. Society of Petroleum Engineers, Mishref, Kuwait, $\mathrm{p} 12$

4. Talebian SH, Beglari A (2019) Application of production datadriven diagnostics workflow for water shut-off candidate selection in tight carbonate field. SN Appl Sci 1(12):1723

5. George BK et al (2012) Challenges and key learning for developing tight carbonate reservoirs. In: Abu Dhabi international petroleum conference and exhibition. Society of Petroleum Engineers, Abu Dhabi, UAE, p 15

6. Economides M, Nolte K (1989) Reservoir stimulation, 3rd edn. Wiley

7. Moghanloo RG, Davudov D, Akita E (2018) Chapter six- formation damage by organic deposition, in formation damage 
during improved oil recovery, B. Yuan and D.A. Wood, Editors. Gulf Professional Publishing, pp 243-273

8. Kartoatmodjo GP et al (2007) Risk-based candidate selection workflow improve acid stimulation success ratio in mature field. In: Asia Pacific oil and gas conference and exhibition. Society of Petroleum Engineers, Jakarta, Indonesia, p 12

9. Wood DA, Yuan B (2018) Chapter fifteen-integrated risks assessment and management of IOR/EOR projects: a formation damage view. In: Formation damage during improved oil recovery, B. Yuan and D.A. Wood, Editors. 2018, Gulf Professional Publishing, pp 587-631

10. Zoback MD (2007) Reservoir geomechanics. Cambridge University Press, Cambridge

11. Nauroy J-FS (2011) Geomechanics applied to the petroleum industry. Editions Technips, Paris

12. Dake LP (1978) Fundamentals of reservoir engineering. Elsevier

13. Chilingar G, Long W (2017) Correlation between porosity and permeability of carbonate rock reservoirs. Energy Sources Part A Recov Utili Environ Effects 39(11):1116-1117

14. Ghabezloo S et al (2009) Effective stress law for the permeability of a limestone. Int J Rock Mech Min Sci 46(2):297-306

15. Nikoosokhan S, Vandamme M, Dangla P (2014) A poromechanical model for coal seams saturated with binary mixtures of $\mathrm{CH} 4$ and CO2.J Mech Phys Solids 71:97-111

16. Ghabezloo S, Sulem J, Saint-Marc J (2009) Evaluation of a permeability-porosity relationship in a low-permeability creeping material using a single transient test. Int J Rock Mech Min Sci 46(4):761-768

17. Allan J, Sun SQ (2003) Controls on recovery factor in fractured reservoirs: lessons learned from 100 fractured fields. In: SPE annual technical conference and exhibition. Society of Petroleum Engineers, Denver, Colorado, p. 18

18. Talebian H, Nikoosokhan S (2020) Coupled hydro-mechanical modeling to improve stimulation candidate selection in a complex carbonate reservoir. In: EAGE 2002 anual conference \& exhibition. European Association of Geoscientists \& Engineers

19. Nnanna E, Osuagwu M, Okereke $O$ (2009) Important considerations in matrix stimulation candidate selection in Niger delta. In: Nigeria annual international conference and exhibition. Society of Petroleum Engineers, Abuja, Nigeria, p 11
20. Gutierrex M, Lewis RW (1998) The role of geomechanics in reservoir simulation. In: SPE/ISRM rock mechanics in petroleum engineering

21. Eaton BA (1969) Fracture gradient prediction and its application in oilfield operations. J Pet Technol 21(10):1353-1360

22. Ghassemi A (2012) A review of some rock mechanics issues in geothermal reservoir development. Geotech Geol Eng 30(3):647-664

23. Blöcher $\mathrm{G}$ et al (2014) Direct and indirect laboratory measurements of poroelastic properties of two consolidated sandstones. Int J Rock Mech Min Sci 67:191-201

24. Yuan $Z$ et al (2013) Casing failure mechanism and characterization under HPHT conditions in South Texas. In: International petroleum technology conference

25. Wang HF (2000) Theory of linear poroelasticity with applications to geomechanics and hydrogeology. Princeton University Press

26. Coussy O (2004) Problems of poroelasticity. In: Poromechanics. Wiley, pp 113-150

27. Homuth S, Götz AE, Sass I (2015) Reservoir characterization of the Upper Jurassic geothermal target formations (Molasse Basin, Germany): role of thermofacies as exploration tool. Geotherm Energy Sci 3:41

28. Harami KK et al (2013) Heterogeneity index and fast screening processes used in a large mature field designed for quick production gains. In: SPE Kuwait oil and gas show and conference. Society of Petroleum Engineers, Kuwait City, Kuwait, p 15

29. Yuan B, Wood DA (2018) Chapter one-overview of formation damage during improved and enhanced oil recovery. In: Yuan B, Wood DA (eds) Formation damage during improved oil recovery. Gulf Professional Publishing, pp 1-20

30. Riyanto $L$ et al (2016) Novel aqueous-based consolidation restores sand control and well productivity: case history from East Malaysia. In: SPE international conference and exhibition on formation damage control. Society of Petroleum Engineers, Lafayette, Louisiana, USA, p 12

Publisher's Note Springer Nature remains neutral with regard to jurisdictional claims in published maps and institutional affiliations. 